\title{
Correlation of dendritic cell maturation and the formation of aggregates of poly-ubiquitinated proteins in the cytosol
}

\author{
Melanie Faßbender $\cdot$ Sylvia Herter $\cdot$ \\ Rafaela Holtappels · Hansjörg Schild
}

Received: 22 February 2008 / Published online: 14 March 2008

(C) Springer-Verlag 2008

\begin{abstract}
Dendritic cells (DCs) are the most powerful antigen presenting cells (APCs) in the immune system. Therefore, they are able to take up antigen by phagocytosis, macropinocytosis or endocytosis, process it in the cytosol and present it to naive $\mathrm{T}$ cells. It is known that presentation of the immunodominant influenza virus nucleoproteinderived CTL epitope is delayed in bone marrow-derived DCs (BMDCs) compared to non-professional APCs. This delay coincided with the formation of transient aggregations of ubiquitinated proteins (DALIS, dendritic cell aggresome-like induced structures), which contain probably defective ribosomal products (DRiPs). DRiPs appear in the cytosol of maturing DCs and macrophages. Normally, DRiPs are degraded rapidly by proteasomes. However, their storage in DALIS delays their degradation. So, it is hypothesized that DALIS can function as antigen depots allowing DCs to coordinate maturation and antigen presentation during their migration to the lymph nodes. Upon inhibition of several pathways among the in signal transduction pathways of DCs, like the phosphatidylinositol 3-kinase (PI3-K) or the mammalian target of Rapamycin (mTOR), the cells show a rendered maturation profile. The formation of DALIS is inhibited in these cells which can be expected to influence antigen processing and presentation.
\end{abstract}

This work was supported by grants from the Deutsche Forschungsgemeinschaft (Sonderforschungsbereich 490, individual projects E6 to H.S. and E3 to R.H.).

M. Faßbender $\cdot$ S. Herter $\cdot$ H. Schild $(\square)$

Institute for Immunology, Johannes Gutenberg-University,

Hochhaus am Augustusplatz 1, 55131 Mainz, Germany

e-mail: schild@uni-mainz.de

R. Holtappels

Institute for Virology, Johannes Gutenberg-University,

Mainz, Germany
Keywords Dendritic cells · DALIS ·

Antigen presentation $\cdot$ CTL activation
Abbreviations
BMDC Bone marrow-derived dendritic cell
CTL Cytotoxic T lymphocytes
DALIS Dendritic cell aggresome-like induced structures
DRiPs Defective ribosomal products
mCMV Murine cytomegalovirus
NP Nucleoprotein
PI3-K Phosphatidylinositol 3-kinase
mTOR Mammalian target of Rapamycin
TLR Toll-like receptor
LPS Lipopolysaccharid

\section{Introduction}

Dendritic cells (DCs) are professional antigen presenting cells (APCs) that play a pivotal role in the initiation of immune responses due to their capacity for antigen presentation and activation of naive cytotoxic $\mathrm{T}$ lymphocytes (CTLs) [1]. In contrast to non-professional APCs, in which early generation and presentation of CTL epitopes is required to allow rapid elimination of the infected cells, the situation for DCs is different. Although viral entry and expression of viral proteins happen very fast, DCs need time to acquire an activated phenotype that allows optimal activation of naïve T cells [2]. During this time the cells undergo changes in their expression levels of surface molecules and they start to secrete proinflammatory cytokines. At the same time an accumulation of protein aggregates, which are polyubiquitinated, can be observed in the cytosol. This phenomenon termed formation of DALIS (dendritic cell aggresome-like induced structures) has been 
described primarily by Hugues Lelouard et al. [3] in dendritic cells and was observed later in macrophages as well [4]. The difference between DALIS and aggresomes can be shown by staining against vimentin which builds a cage around normal aggresomes but not around DALIS. In addition, nocodazole can be used to differentiate between DALIS and aggresomes because it interferes with the structure and function of microtubules which are important for aggresome formation. Furthermore, DALIS formation is a transient event starting $6-8 \mathrm{~h}$ after activation of DCs with TLR-ligands or viruses and reaching a maximum at $15 \mathrm{~h}$. After around $20-24 \mathrm{~h}$ no DALIS can be found anymore. At this time, DCs are thought to have reached the draining lymph nodes and have expressed costimulatory molecules. So, it was hypothesized that DALIS can function as antigen depots which delay the antigen presentation in DCs until a proper maturation status has been achieved which ensures efficient activation of $\mathrm{T}$ cells. In line with this hypothesis it has been shown that the presentation of, e.g. the immunodominant influenza virus nucleoprotein (NP)-derived CTL epitope is delayed in BMDCs compared to non-professional APCs [5], and this delay coincides with the formation of DALIS.

Little is known about the mechanisms leading to the formation of DALIS. However, their appearance is tightly linked to the activation of DCs. The main signalling cascades in DCs during maturation are more or less well defined. The phosphatidylinositol 3-kinase (PI3-K) plays a central role in DC activation. This molecule is a lipid kinase and is present in every eukaryotic cell. It affects cell survival, proliferation, migration and differentiation. Its main function is the production of the second messenger phosphatidylinositol-3,4,5-trisphosphate (PIP3) which catalyzes downstream activation of other molecules like Akt and the mammalian target of Rapamycin (mTOR) [6, 7]. mTOR belongs to the family of the phosphatidylinositol 3-kinases. This serine/threonine kinase is involved in cell proliferation and differentiation as well as in the translation of proteins. Both molecules are essential for DC maturation and antigen processing and presentation. We will discuss in this article recent developments shedding light on the linkage of signalling pathways in DCs and the formation of DALIS.

\section{DALIS formation and activation of cytotoxic T cells}

Cytotoxic T cell (CTL) activation requires the presentation of peptides bound to MHC class I molecules on the surface of antigen presenting cells. These peptides are generated in the cytosol and can be derived from extra- or intracellular sources. A large fraction of newly synthesized proteins are defective ribosomal products (DRiPs) [8-10] due to mistakes in their sequences, in folding or post-translational processes. In non-professional APCs these DRiPs are degraded rapidly by proteasomes [11] and the resulting peptides are presented by MHC class I molecules to CTL. However, in DCs DRiPs are poly-ubiquitinated and stored transiently in DALIS which delays their degradation [12]. So, it was hypothesized that DALIS can function as antigen depots allowing DCs to delay antigen presentation until they reach a mature phenotype and the draining lymph nodes where they meet naïve $\mathrm{CD} 8$ positive $\mathrm{T}$ cells for activation. In line with this it was observed that the nucleoprotein of the influenza virus can be found in DALIS of virus infected DCs [5]. Interestingly, the kinetic of the MHCrestricted presentation of nucleoprotein derived peptides correlated with the upregulation of MHC class I molecules as well as costimulatory molecules and resulted in an efficient recognition of infected DCs by influenza virus NPspecific CTL only around $12 \mathrm{~h}$ after infection. In contrast, non-professional APC, like fibroblasts, which lack DALIS formation are detected by NP-specific CTL already $2-3 \mathrm{~h}$ after infection [5]. Thus, the coordination of the events resulting in DC maturation and peptide presentation might be an important feature for the activation of naïve CD8 positive $T$ cells because antigen recognition in the absence of costimulation results in the induction of T cell anergy.

\section{Inhibition of PI3-kinase interferes with DC maturation and DALIS formation}

Toll-like receptor (TLR)-mediated maturation of DCs involves several mitogen-activated protein kinase (MAPK) pathways: the extracellular signal-regulated protein kinase (ERK), the p38 stress-activated protein kinase (p38), and the c-Jun N-terminal kinase (JNK). Besides, there exist additional signal transduction pathways mediating cellular responses to external stimuli. One of those pathways is the PI3-K/Akt pathway. Bhattacharyya et al. [13] reported a reduction of TNF- $\alpha$ and IL-12 production after treatment of DCs with the PI3-K inhibitor Ly294002. It also could be shown that after inhibition of PI3-K the expression of the costimulatory molecules is decreased as well as the secretion of proinflammatory cytokines. In parallel, inhibition of MEK-1 by the specific inhibitor PD98059 does not lead to alterations in the maturation of the DCs (Fig. 1).

Although it is speculated that DALIS play a role as antigen depots, the exact function of these aggregates and the mechanism of their formation in maturing DCs and macrophages remains unclear. In order to investigate a direct connection between DALIS formation and stimulation of BMDCs by TLR ligands, the effects of several inhibitors of the TLR signalling pathways on DALIS formation were analysed. Immature BMDCs were found to show little or no DALIS formation (Fig. 2), whereas LPS induces DALIS 
Fig. 1 The PI3-K-inhibitor Ly294002 influences DC maturation. BMDCs were treated with the indicated concentrations of the inhibitors Ly294002 $(\mathbf{a}, \mathbf{b})$ or PD98059 (c, d). After $1 \mathrm{~h}$, the cells were stimulated with $100 \mathrm{ng} / \mathrm{ml}$ LPS or $50 \mu \mathrm{g} / \mathrm{ml}$ poly (I:C) or were infected with $1 \mathrm{PFU} /$ cell of influenza virus A $\mathrm{PR} / 8 / 34$. Eighteen hours later, IL-6 was measured in the culture supernatants by using sandwichELISA $(\mathbf{a}, \mathbf{c})$. The cells were stained with anti-CD80-FITC and anti-CD86-PE antibodies and analysed by FACS (b, d). To directly compare the inhibitor effects on the different stimuli, data were normalized to the data obtained for accordingly stimulated cells in absence of inhibitor treatment
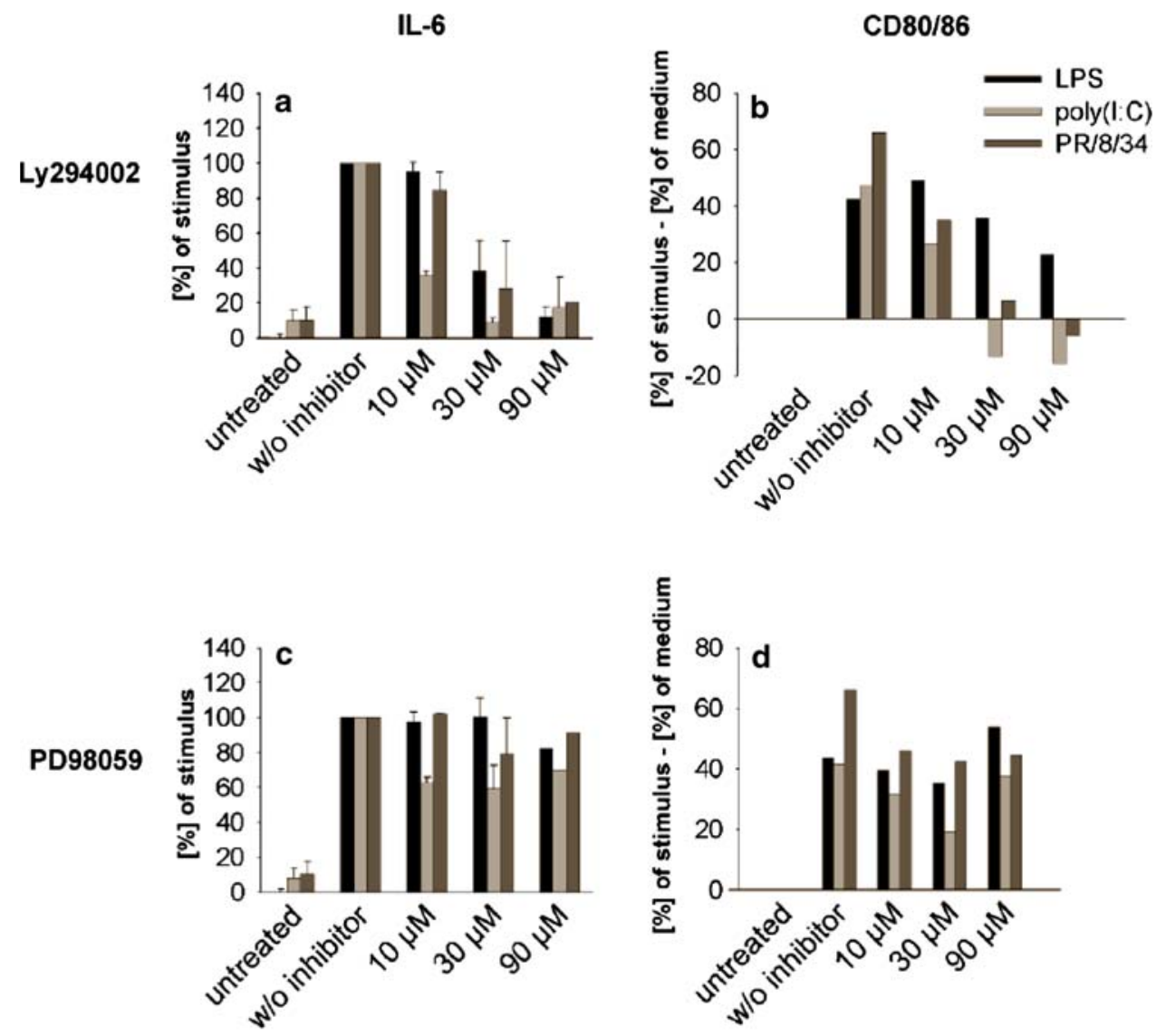

inhibitor concentration formation. The induction of DALIS is not influenced by inhibitors of the various MAPK pathways, but is strongly reduced by the PI3-K inhibitor Ly294002.

$\mathrm{PI} 3-\mathrm{K}$ is known to be involved in cell differentiation, metabolism, proliferation and survival. Regarding the function of DCs it has been shown recently that DCs of $\mathrm{PI} 3-\mathrm{K}^{-1-}$ mice have a reduced migratory capacity under inflammatory conditions [14]. Furthermore, inhibition of the PI3-K/Akt pathway led to decreased survival of human monocyte-derived DCs as well as decreased upregulation of costimulatory molecules after activation [15].

\section{mTOR inhibition results in total impairment of DALIS formation}

The targets of Rapamycin (TOR) are cytoplasmic protein kinases which are responsible for the initiation of translation, ribosome biosynthesis and the transcription of many enzymes. Rapamycin, the potent inhibitor for the mammalian target of Rapamycin (mTOR), is previously described as immunosuppressive drug [16]. This macrolide antibiotic, produced by Streptomyces hygroscopicus, was introduced to prevent allograft rejection. With its homology to PI3-K, mTOR belongs to the lipid kinase family. Its inhibition is known to block $\mathrm{T}$ cell proliferation by prevention of IL-2 production and induces cell cycle arrest at $\mathrm{G}_{1}$ phase [17]. In the cell, Rapamycin binds to FKBPs (FK506 binding proteins), a specific family of cytosolic proteins (immunophilins) $[17,18]$. It is known that mTOR inhibition in APCs during the time of culturing attenuates cytokine production and surface marker expression as well as it prevents macropinocytosis and receptor mediated endocytosis [16]. Additionally, in higher doses Rapamycin induces apoptosis. After inhibition of mTOR in immature BMDCs the cells show diminished IL- 6 but no alteration in the IL-12p40 production (Fig. 3). Furthermore, mTOR inhibition in BMDCs leads to complete abrogation of DALIS formation even at low doses of Rapamycin.

\section{Future aspects}

Recently it has also been shown by Lelouard et al. [19] that $\mathrm{PI} 3-\mathrm{K}$ and downstream mTOR signalling is important for protein translation and that DALIS formation is closely 
Fig. 2 The PI3-K-inhibitor Ly294002 prevents DALIS formation. BMDCs were left untreated (a), stimulated with $100 \mathrm{ng} / \mathrm{ml}$ LPS (b), or treated prior to LPS stimulation with $30 \mu \mathrm{M}$ PD98059 (c) or $30 \mu \mathrm{M}$ Ly294002 (d). Thereafter, cells were labeled with biotinylated FK2 antibody (specific for mono- and polyubiquitinated proteins) and streptavidin-FITC to visualize DALIS (green fluorescence) as well as with DAPI to stain the cell nuclei (blue)

Scale bars: $20 \mu \mathrm{m}$
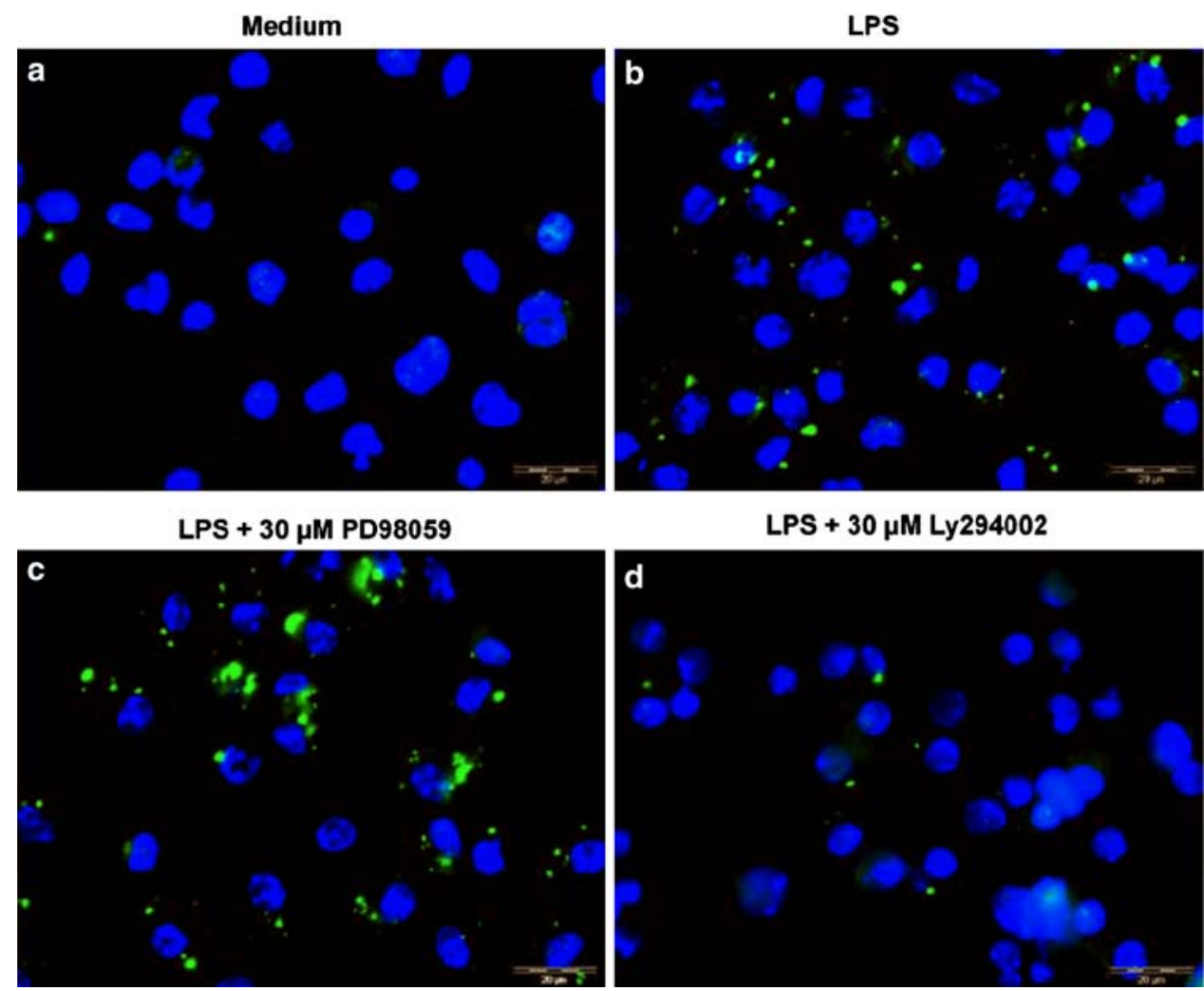

a

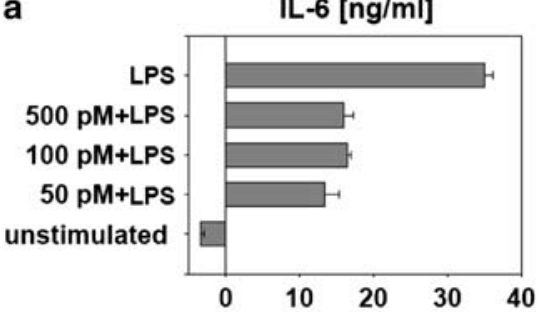

IL-12p40 [ng/ml]

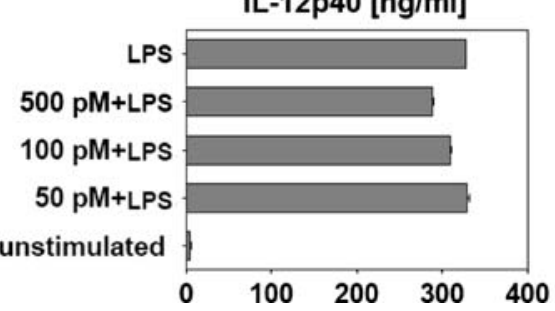

Fig. 3 mTOR inhibition leads to diminished DALIS formation. BMDCs were stimulated with LPS $(100 \mathrm{ng} / \mathrm{ml})$ or left untreated. Additionally, cells were treated with Rapamycin in the indicated concentrations 30 min before LPS activation. The secretion of IL- 6 and IL-12p40 after $20 \mathrm{~h}$ of culture was analysed by using sandwich-ELISA (a). To analyse DALIS formation, cells were stimulated with LPS (100 ng/ml), left un-

linked to protein translation as well. It is speculated that DALIS represent a cellular response to stress associated to dramatic changes in protein synthesis due to DC activation which is accompanied by the accumulation of DRiPs. It was shown recently that cells lacking DALIS formation, b

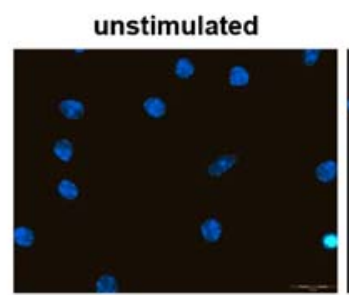

Rapamycin 500 pM
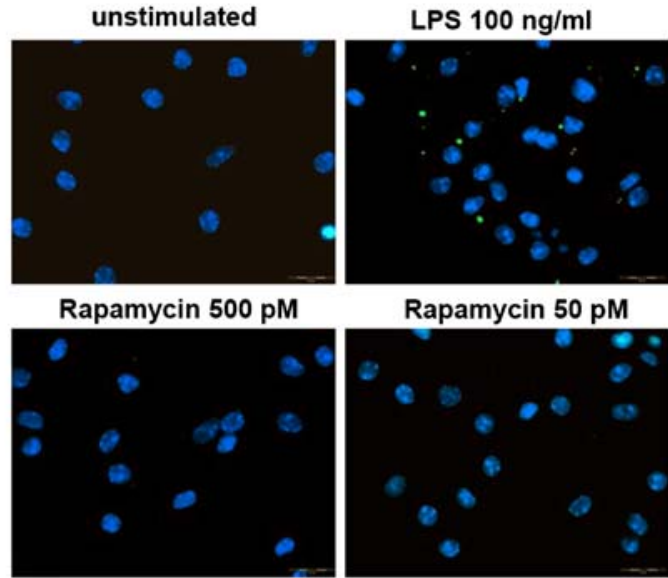

Rapamycin 50 pM

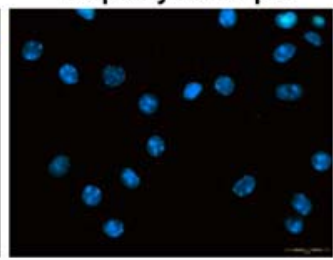

treated or were pretreated with the indicated concentrations of Rapamycin before LPS activation (b). Thereafter, cells were labeled with biotinylated FK2 antibody and streptavidin-FITC to visualize DALIS (green fluorescence) as well as with DAPI to stain the cell nuclei (blue). Scale bars: $20 \mu \mathrm{m}$

e.g. EL-4 tumor cells or fibroblasts display an accelerated presentation of influenza virus-derived peptides after infection when compared to influenza virus infected BMDCs [5]. It will now be interesting to compare the antigen presentation kinetics of influenza virus-infected Ly294002- 
treated BMDCs, where activation and DALIS formation is inhibited and resembles those of EL-4 cells, with untreated virus-infected BMDCs.

Notably, murine cytomegalovirus (mCMV)-infected BMDCs do not show DALIS formation (Fassbender et al., manuscript in preparation). This virus contains ingenious strategies to hide inside its host-cell without being recognized. Therefore it expresses so-called immunoevasins that make sure that the MHC-peptide complexes do not reach the cell surface or lead them directly to the proteasome for degradation [20, 21]. It will be interesting to analyse if the lack of DALIS formation after mCMV infection contributes to the strategies of mCMV to escape from efficient recognition by the host immune system. As the signalling events accompanying DALIS formation are beginning to unfold it might be soon possible to modulate DALIS formation in professional antigen presenting cells like DCs without affecting their maturation. This might allow finally elucidating the role of DALIS during processing and presentation of MHC class I ligands and thus influencing the role of DCs in the induction of adaptive immune responses.

\section{References}

1. Mellman I, Steinman RM (2001) Dendritic cells: specialized and regulated antigen processing machines. Cell 106:255-258

2. Guermonprez P, Valladeau J, Zitvogel L, Thery C, Amigorena S (2002) Antigen presentation and T cell stimulation by dendritic cells. Annu Rev Immunol 20:621-667

3. Lelouard H, Gatti E, Cappello F, Gresser O, Camosseto V, Pierre $P$ (2002) Transient aggregation of ubiquitinated proteins during dendritic cell maturation. Nature 417:177-182

4. Canadien V, Tan T, Zilber R, Szeto J, Perrin AJ, Brumell JH (2005) Cutting edge: microbial products elicit formation of dendritic cell aggresome-like induced structures in macrophages. J Immunol 174:2471-2475

5. Herter S, Osterloh P, Hilf N, Rechtsteiner G, Hohfeld J, Rammensee HG, Schild H (2005) Dendritic cell aggresome-like-induced structure formation and delayed antigen presentation coincide in influenza virus-infected dendritic cells. J Immunol 175:891-898

6. Cantley LC (2002) The phosphoinositide 3-kinase pathway. Science 296:1655

7. Reuben JS (2006) Ras, PI(3)K and mTOR signalling controls tumor cell growth. Nature 441:424-430
8. Yewdell JW, Schubert U, Bennink JR (2001) At the crossroads of cell biology and immunology: DRiPs and other sources of peptide ligands for MHC class I molecules. J Cell Sci 114:845-851

9. Yewdell J (2002) To DRiP or not to DRiP: generating peptide ligands for MHC class I molecules from biosynthesized proteins. Mol Immunol 39:139-146

10. Yewdell JW, Anton LC, Bennink JR (1996) Defective ribosomal products (DRiPs): a major source of antigenic peptides for MHC class I molecules? J Immunol 157:1823-1826

11. Schubert U, Anton LC, Gibbs J, Norbury CC, Yewdell JW, Bennink JR (2000) Rapid degradation of a large fraction of newly synthesized proteins by proteasomes. Nature 404:770-774

12. Lelouard H, Ferrand V, Marguet D, Bania J, Camosseto V, David A, Gatti E, Pierre P (2004) Dendritic cell aggresome-like induced structures are dedicated areas for ubiquitination and storage of newly synthesized defective proteins. J Cell Biol 164:667-675

13. Bhattacharyya S, Sen P, Wallet M, Long B, Baldwin AS Jr., Tisch $R$ (2004) Immunoregulation of dendritic cells by IL-10 is mediated through suppression of the PI3K/Akt pathway and of IkappaB kinase activity. Blood 104:1100-1109

14. Del Prete A, Vermi W, Dander E, Otero K, Barberis L, Luini W, Bernasconi S, Sironi M, Santoro A, Garlanda C, Facchetti F, Wymann MP, Vecchi A, Hirsch E, Mantovani A, Sozzani S (2004) Defective dendritic cell migration and activation of adaptive immunity in PI3Kgamma-deficient mice. EMBO J 23:3505-3515

15. Ardeshna KM, Pizzey AR, Devereux S, Khwaja A (2000) The PI3 kinase, p38 SAP kinase, and NF-kappaB signal transduction pathways are involved in the survival and maturation of Lipopolysaccharide-stimulated human monocyte-derived dendritic cells. Blood 96:1039-1046

16. Hackstein H (2002) Rapamycin inhibits macropinocytosis and mannose receptor-mediated endocytosis by BMDC. Blood 100(3): 1084

17. Morice WG (1993) Rapamycin inhibition of interleukin-2-dependent $\mathrm{p} 33 \mathrm{cdk} 2$ and $\mathrm{p} 34 \mathrm{cdc} 2$ kinase activation in T lymphocytes. J Biol Chem 268(30):22737-22745

18. Sehgal SN (1998) Rapamune ${ }^{\circledR}$ (RAPA, rapamycin, sirolimus): mechanism of action immunosuppressive effect results from blockade of signal transduction and inhibition of cell cycle progression. Clin Biochem 31(5):335-340

19. Lelouard H (2007) Regulation of translation is required for dendritic cell function and survival during activation. JCB 179(7):1427-1439

20. Reddehase MJ (2002) Antigens and immunoevasins: opponents in cytomegalovirus immune surveillance. Nat Rev Immunol 2:831844

21. Wagner M, Gutermann A, Podlech J, Reddehase MJ, Koszinowski UH (2002) Major histocompatibility complex class I allele-specific cooperative and competitive interactions between immune evasion proteins of cytomegalovirus. J Exp Med 196:805-816 